FORMATLON Formation emploi

Revue française de sciences sociales

105 | janvier-mars 2009

Pêle-mêle

\title{
Les distorsions diplômes/qualifications : l'exemple des techniciens et agents de maitrise de type industriel
}

Diskrepanzen zwischen Berufsabschlüssen und Qualifizierungen am Beispiel von Industrietechnikern und-meistern

Distortions between diplomas and qualifications: the example of industrial

technicians and supervisors

Las distorsiones diplomas/calificaciones : el ejemplo de los técnicos y

supervisores de tipo industrial

\section{Paul Santelmann}

\section{OpenEdition}

Journals

Édition électronique

URL : http://journals.openedition.org/formationemploi/1834

DOI : 10.4000/formationemploi.1834

ISSN : 2107-0946

Éditeur

La Documentation française

Édition imprimée

Date de publication : 1 mars 2009

Pagination : 53-65

ISSN : 0759-6340

Référence électronique

Paul Santelmann, «Les distorsions diplômes/qualifications : l'exemple des techniciens et agents de maîtrise de type industriel », Formation emploi [En ligne], 105 | janvier-mars 2009, mis en ligne le 01 mars 2011, consulté le 30 octobre 2020. URL : http://journals.openedition.org/formationemploi/1834 ; DOI : https://doi.org/10.4000/formationemploi.1834 
Débat

\section{Les distorsions diplômes/ qualifications : I'exemple}

\section{des techniciens et agents de maîtrise de type industriel}

Par Paul Santelmann*

La complexification du travail n'a été envisagée qu'en termes de montée en niveau de formation initiale. Cela a accentué la sélectivité de l'accès aux emplois intermédiaires (déclassement) et réduit l'espace promotionnel des ouvriers et employés expérimentés.

Cette analyse sera débattue dans notre prochain numéro.

De nombreux travaux soulignent l'écart entre l'évolution de la carte des qualifications professionnelles et celle des diplômes : «En 30 ans, le paysage éducatif et celui de l'emploi se sont totalement transformés alors que la nomenclature initiale n'a pas été revue. (...) Plus important peut-être, l'architecture des catégories d'emplois de référence est celle des années 70 , soit celle d'une forte division du travail, de la séparation entre fonctions de production, conception, contrôle, une conception industrielle tayloriste. L'évolution de l'emploi, en particulier la tertiarisation, conduit au développement d'activités professionnelles et d'emplois qui s'inscrivent mal dans les dispositifs classiques de définition des qualifications, classifications, catégories qui sont à la base de la correspondance de cette nomenclature. (...) Paradoxalement, c'est pour "mieux coller aux réalités de
* Paul Santelmann est chef du service prospective à la Direction générale de l'AFPA (Association nationale pour la formation professionnelle des adultes). II est chargé d'enseignement à l'université de Paris I (La Sorbonne) et au CNAM (Conservatoire national des arts et métiers). II est notamment l'auteur de : La formation professionnelle continue, collection "Les études », La Documentation française, 2006 ; De l'efficacité en formation continue, Éditions Liaisons, collection « Entreprise \& Carrières », 2004 ; Qualification ou compétences, en finir avec la notion d'emplois non qualifiés, Éditions Liaisons, collection "Entreprise \& Carrières », 2002 ; La formation professionnelle, nouveau droit de l'homme?, Éditions Gallimard, collection "Folio actuel, Le Monde», 2001 
l'emploi" que le système de formation s'est enrichi de nouveaux diplômes et formations complémentaires... » (Dauty, Lemistre et Vincens, 2006). Entre 1982 et 2002, le nombre de cadres est en effet passé de 2,7 millions à 4,3 millions, celui des techniciens et des professions intermédiaires de 3,3 millions à 4,6 millions. Le poids de ces deux catégories professionnelles dans l'emploi global a évolué de $27,6 \%$ à $36,9 \%$.

Cette évolution illustre l'importance des transformations des organisations du travail et interpelle l'École, qui a longtemps été subordonnée aux représentations liées à une division sociale et technique du travail considérée comme immuable. Or la complexification technico-organisationnelle des contenus de travail n'a été interprétée qu'à l'aune de la montée en niveau des objectifs de la formation initiale. Ainsi les instances chargées de la conception des diplômes professionnels ont principalement relayé les logiques d'accumulation de savoirs initiaux et d'allongement des études au détriment d'une réagrégation des voies générale, technologique et professionnelle. L'enseignement professionnel de niveaux $\mathrm{V}$ et IV (respectivement formation de niveaux CAP-BEP - certificat d'aptitude professionnelle et brevet d'études professionnelles - et baccalauréat) a ainsi été maintenu comme voie de relégation, et la façon dont la formation continue pouvait accompagner les salariés dans les évolutions du travail a été occultée. Ces choix ont considérablement accentué la sélectivité de l'accès aux emplois intermédiaires (avec les effets de déclassement constatés depuis) et réduit l'espace promotionnel des ouvriers et employés qualifiés expérimentés. Cela sans répondre aux attentes des entreprises qui sont confrontées à des difficultés de recrutement dans des fonctions mal identifiées car échappant aux catégorisations traditionnelles de l'emploi.

On assiste à un renouvellement important des titulaires des emplois intermédiaires (départ en retraite des papys boomers (CGP, 2002 ; CAS, 2007)) ; dans ce contexte, nous avons choisi d'examiner le cas des techniciens et agents de maîtrise (TAM) de type industriel, au regard des objectifs des BTS et DUT (respectivement brevet de technicien supérieur et diplôme universitaire de technologie), comme illustration du décalage entre les multiples ambitions des

\section{Encadré 1}

L'enseignement supérieur court dans les spécialités industrielles

Dans les spécialités industrielles, les diplômés des sections de techniciens supérieurs (STS) étaient 23242 en $2006(*)$, pour environ $12780\left({ }^{* *}\right)$ diplômés des instituts universitaires de technologie (IUT). Si on ajoute les maigres effectifs des licences professionnelles 16637 dans les spécialités de la production) et sachant qu'environ $20 \%$ des BTS et $60 \%$ des DUT poursuivent leurs études, la production globale de diplômés de niveau bac +2 ou 3 pour les besoins du marché du travail est d'environ 26300 diplômés 14000 DUT, 18000 BTS, 4300 licences pro). L'apport de la formation continue qualifiante au niveau III (inclues les formations de I'AFPA - Association nationale pour la formation professionnelle des adultes) correspond à environ 6500 diplômés par an.

$\left(^{*}\right)$ : «Résultats des brevets de techniciens supérieurs session $2006 »$ - DEP - Note d'information $\mathrm{n}^{\circ} 19-2007$. $(* *)$ : «La réussite en DUT » - DEP - Note d'information $n^{\circ} 18-2007$.

politiques de validation - supposées favoriser l'accès à l'emploi - et les carrières professionnelles ascendantes d'une part et la structure des qualifications professionnelles d'autre part. Ce questionnement permettra aussi de réinterroger des débats antérieurs relatifs à la concomitance de la déqualification supposée du travail ouvrier et de l'émergence des techniciens (Tanguy, 1986 ; Stroobants, 1993) et à la façon dont les diplômes professionnels relayent ou amplifient telle ou telle hypothèse.

Nous aborderons plus particulièrement les dominantes fonctionnelles et techniques des TAM, telles qu'elles sont retranscrites dans les différentes nomenclatures d'emploi, ainsi que le niveau d'études des salariés concernés. Nous mobiliserons les questionnements des chercheurs confrontés à l'interprétation des mutations du travail industriel et aux décalages entre les profils des jeunes diplômés et les 
attentes des employeurs. Enfin nous tenterons de cerner les tensions relatives à la conception des diplômes à visée professionnelle.

\section{L'IMPORTANCE DES FONCTIONS DE PRODUCTION CHEZ LES TECH- NICIENS ET AGENTS DE MAÎTRISE}

Pour l'INSEE, la catégorie socioprofessionnelle des techniciens et agents de maîtrise (TAM) regroupe les salariés qui appliquent, dans leur activité, des connaissances ou pratiques technologiques et sont classés agents de maîtrise ou techniciens dans les conventions collectives. Dans le contexte de transformation de la carte des qualifications professionnelles, les techniciens et agents de maîtrise du système industriel (un million d'emplois) exercent d'abord des fonctions liées à la production (70\%). Quant aux fonctions d'encadrement et aux fonctions d'études, elles ne pèsent respectivement que $20,4 \%$ et $5,6 \%$ des emplois (voir tableau 1).

${ }^{1}$ Les données relatives aux TAM sont issues des portraits statistiques de la DARES (Familles professionnelles - 20 ans de métiers - octobre 2004).
Les nomenclatures d'emploi (voir encadré 2) attestent du caractère pour le moins brouillé de ce niveau d'emploi par rapport aux niveaux des diplômes. L'analyse des profils des TAM est d'ailleurs parlante puisque $53 \%$ d'entre eux (près de 540000 ) ont un niveau infra-bac et n'ont pas un profil socio-culturel distinct des ouvriers qualifiés. On notera que l'écart quantitatif entre les ouvriers qualifiés et les TAM est de 2 pour 1 (voir tableau 2).

Sans entrer dans une comparaison fine entre les ouvriers qualifiés et les TAM, on observe de nombreuses porosités entre ces deux groupes. L'INSEE apporte d'ailleurs de nombreuses nuances concernant la catégorisation des techniciens dans les conventions collectives, en précisant notamment que : «dans certaines conventions collectives, notamment chimie et IAA, les aides techniciens, aides de laboratoires, aides techniques... sont classés avec les techniciens alors que leur niveau correspond à celui des ouvriers qualifiés (...). L'expression "Technicien d'atelier", dans la mécanique, la construction électrique, et électronique, la sidérurgie... désigne le niveau supérieur de la filière "ouvrier" ". Si les qualifications ouvrières sont plutôt concentrées dans le secteur industriel au sens strict et traditionnel, les TAM industriels sont beaucoup plus diffusés et se répartissent dans une vingtaine de grands secteurs. $40 \%$ des TAM sont d'ailleurs ventilés dans des

Tableau 1

Répartition fonctionnelle des Techniciens et agents de maîtrise (TAM)

\begin{tabular}{|c|c|c|c|c|c|c|}
\hline & $\begin{array}{l}\text { TAM des } \\
\text { industries } \\
\text { mécaniques }\end{array}$ & $\begin{array}{l}\text { TAM des } \\
\text { industries } \\
\text { de process }\end{array}$ & $\begin{array}{c}\text { TAM } \\
\text { maintenance } \\
\text { et } \\
\text { organisation }\end{array}$ & $\begin{array}{c}\text { TAM } \\
\text { industries } \\
\text { légères et } \\
\text { graphiques }\end{array}$ & $\begin{array}{c}\text { TAM } \\
\text { électricité, } \\
\text { électronique, } \\
\text { maintenance }\end{array}$ & TOTAL \\
\hline $\begin{array}{l}\text { Techniciens } \\
\text { de production }\end{array}$ & 93600 & 219460 & 233790 & 70230 & 97890 & $\begin{array}{c}714970 \\
(70,2 \%)\end{array}$ \\
\hline Fonctions d'études & 38280 & & & & 18830 & $\begin{array}{l}57110 \\
(5,6 \%)\end{array}$ \\
\hline $\begin{array}{l}\text { Agents } \\
\text { d'encadrement }\end{array}$ & 111330 & 77230 & & & 19510 & $\begin{array}{r}208070 \\
(20,4 \%)\end{array}$ \\
\hline Organisation & & & 38090 & & & $\begin{array}{r}38090 \\
(3,7 \%)\end{array}$ \\
\hline TOTAL & 243210 & 296690 & 271880 & 70230 & 136230 & $\begin{array}{r}1018240 \\
(100 \%)\end{array}$ \\
\hline
\end{tabular}

Source : portraits statistiques 1982-2002 des FAP, Dares, octobre 2004. 


\section{Encadré 2}

\section{Les nomenclatures utilisées}

L'INSEE (*) a réparti les TAM (techniciens et agents de maîtrise) en deux catégories (47 et 48). La catégorie 47 des techniciens industriels recouvre plusieurs champs fonctionnels : les études, la recherchedéveloppement, et les méthodes, la fabrication et le contrôle-qualité et les fonctions connexes à la production (ordonnancement-programmation-logistique, maintenance, environnement). Les principaux emplois de techniciens industriels comportent une dimension "production et intervention technique " essentielle. Il en va ainsi de l'activité des techniciens de fabrication et de contrôle-qualité en construction mécanique et travail des métaux (474c), en électricité, électromécanique et électronique (473c) et des industries de transformation (475 b). Quant aux agents de maîtrise, regroupés dans la catégorie 48, s'ils exercent une fonction d'encadrement, ils possèdent aussi des compétences de production manifestes. Ainsi les agents de maîtrise ou des techniciens des ateliers de fabrication mécanique (483a) "conseillent sur les méthodes opératoires, éventuellement interviennent directement dans la production ", les agents de maîtrise en maintenance, installation en électricité et électronique (486b) ou les agents de maîtrise en maintenance, installation en électromécanique (486c) sont chargés de répartir les tâches entre les membres de l'équipe et de "leur apporter une assistance technique dans les cas difficiles ».

Le répertoire opérationnel des métiers et des emplois (ROME), élaboré par l'ANPE (Agence nationale pour l'emploi), précise les compétences et les savoir-faire des métiers au-delà des appellations courantes et a défini trois types de techniciens industriels:

1) Les techniciens de préparation de la production, où l'on retrouve les fonctions de méthodes, d'organisation, d'études et de R\&D - recherche et développement (code $521 \rightarrow 8$ fiches métiers) ;

2) Les techniciens de fabrication et de contrôle (code $522 \rightarrow 11$ fiches métiers);

3) Les techniciens d'installation et de maintenance (code $523 \rightarrow 10$ fiches métiers).

Cette approche permet de distinguer plus nettement les techniciens de production des techniciens d'études mais ne permet pas de les dénombrer. Sous cet angle, les familles professionnelles (FAP) créées par la DARES ont eu le mérite de croiser la nomenclature PCS (professions et catégories sociales) de I'INSEE avec le ROME afin d'atténuer la logique statutaire de l'approche de l'INSEE mais également la logique sectorielle qui démultiplie les spécialisations dans un même corpus technique et minore la dimension transversale (intersectorielle) de nombreux métiers. Le découpage des TAM industriels en FAP s'est cependant heurté à de nombreux dilemmes : fallait-il privilégier un découpage technologique (mécanique, électricité/électronique, informatique industrielle, etc.), un découpage sectoriel (industrie lourde, industrie légère, industrie de process,...) ou un découpage fonctionnel (encadrement, études, maintenance, organisation, etc.) ? Le choix de privilégier un découpage technologique et sectoriel en cinq familles (mécanique, process, maintenance et organisation, industries légères et graphiques, électricité/ électronique) n'a pas éliminé pour autant l'approche fonctionnelle déclinée dans chaque FAP. Mais cette dimension, qui renvoie aux qualifications, demeure secondaire dans l'usage courant des FAP alors que nous l'avons mise en exergue dans cette contribution.

Dans un marché de l'emploi beaucoup plus instable qu'il y a quarante ans, le traitement des statistiques de l'emploi selon la nomenclature des FAP permet une approche plus dynamique des recompositions de l'emploi et des mobilités. Les FAP se sont ainsi imposées comme critère d'appréciation des évolutions de l'emploi pour les systèmes de formation, les politiques d'emploi et les analyses prospectives ( ${ }^{\star *}$ ). Encore fautil accompagner ces éclairages par des analyses qualitatives des contenus de travail.

$\left({ }^{*}\right)$ : Toutes les définitions qui suivent sont issues de la nomenclature PCS (professions et catégories sociales).

(**) : Les métiers à 2015, Rapport du groupe « prospective des métiers et des qualifications », 2007, La Documentation française. 
Tableau 2

Comparaison des effectifs ouvriers qualifiés et techniciens et agents de maîtrise

\begin{tabular}{|l|r|r|r|r|r|r|}
\hline \multicolumn{1}{|c|}{$\begin{array}{c}\text { Données } \\
2002-\text { DARES }\end{array}$} & \multicolumn{1}{|c|}{$\begin{array}{c}\text { Industries } \\
\text { mécaniques }\end{array}$} & \multicolumn{1}{|c|}{$\begin{array}{c}\text { Industries } \\
\text { de process }\end{array}$} & $\begin{array}{c}\text { Maintenance } \\
\text { et } \\
\text { organisation }\end{array}$ & $\begin{array}{c}\text { Industries } \\
\text { légères et } \\
\text { graphiques }\end{array}$ & $\begin{array}{l}\text { Électricité et } \\
\text { électronique }\end{array}$ & TOTAL \\
\hline $\begin{array}{l}\text { Ouvriers qualifiés de } \\
\text { l'industrie }\end{array}$ & 468200 & 445900 & 354800 & 98700 & 83200 & 1450800 \\
\hline TAM de la production & 93623 & 219459 & 233793 & 70228 & 97886 & 714989 \\
\hline TOTAL & 561822 & 665359 & 588593 & 167928 & 181086 & 2165789 \\
\hline
\end{tabular}

Source : portraits statistiques 1982-2002 des FAP, Dares, octobre 2004.

secteurs qui ne correspondent pas à leur cœur technologique. Les secteurs "pluri-techniques» (qui emploient au moins trois grandes spécialités de TAM) sont : le secteur équipement mécanique qui emploie $7 \%$ des effectifs de TAM, la construction qui emploie $6 \%$ des effectifs et le secteur conseil/ assistance qui emploie $5 \%$ des effectifs. Dans le seul champ de la production et de l'encadrement, les TAM exercent près d'une centaine de spécialisations différentes ${ }^{2}$. Cette variété renvoie à des technologies, à des différenciations sectorielles et des nouvelles organisations du travail qui ont été appropriées par de nombreux salariés infra-bac. On mesure ainsi, compte tenu de la grande variété des conventions collectives des industries mais également des services ou de la construction, qu'il peut y avoir de nombreux recoupements entre les ouvriers qualifiés et les $\mathrm{TAM}^{3}$.

\section{LES TRANSFORMATIONS DU TRAVAIL INDUSTRIEL}

Les caractéristiques des activités des TAM ne sont compréhensibles qu'en rapport avec la centralité du système industriel dans l'économie. Celui-ci ne se

\footnotetext{
2 Nomenclature PCS de l'INSEE.

${ }^{3}$ L'analyse des TAM interroge également le périmètre statistique de ces emplois dans une économie où l'émergence des services s'est confondue avec l'extension d'un secteur tertiaire assimilé à un univers «non technologique». Ainsi les intérimaires ou les informaticiens de l'industrie sont comptabilisés dans les services, l'informatique elle-même étant considérée comme une excroissance « tertiaire » distincte des technologies industrielles.
}

résume plus à une simple ligne de production de produits mais s'est diffusé comme élément consubstantiel de fonctionnement de nombreux services. Cette réalité définit à la fois la carte des compétences nécessaires à l'exercice de cette famille professionnelle et celle des mobilités professionnelles (transférabilité des compétences). La «machinerie» demeure donc omniprésente dans le quotidien et les loisirs (univers domestique, ascenseurs, véhicules, ordinateurs, manèges de foire, construction, appareillage des hôpitaux, etc.): "Dans la sphère des services, il apparaît, de plus, qu'au-delà de la production de services destinés à l'industrie, de nombreux services ont des caractéristiques qui, habituellement, sont associées aux biens industriels: ils peuvent être stockables, exportables, dissociables $d u$ producteur et de l'utilisateur voire directement liés à la notion de transformation physique. » (Flacher et Pelletan, 2007)

La fiabilité technique de cet ensemble est devenue essentielle (sécurité, environnement, qualité des services) et témoigne de deux processus : une diffusion des savoirs techniques dans tous les univers du travail (ce qui relativise d'ailleurs les thèses sur la tertiarisation/abstraction de l'économie) et la pénétration des normes qualité dans l'industrie qui bousculent l'hyper-division du travail : «Les activités qui concourent à cette efficacité sont celles qui s'inscrivent le plus directement dans la tradition industrielle. Mais ce sont aussi celles où, globalement, le travail s'éloigne le plus résolument des modèles tayloriens. » (Veltz, 2000).

Les TAM sont ainsi au cœur d'une des principales contradictions que le système industriel doit gérer 
depuis quelques décennies : la diminution des lignes hiérarchiques et l'allégement de l'encadrement traditionnel (chefs d'atelier) dans les usines et, au-delà, le développement de nombreuses fonctions d'intervention liées aux techniques sophistiquées de l'industrie. Les TAM illustrent d'ailleurs cette mutation puisque s'y retrouvent précisément les cadres intermédiaires (en déclin) et les techniciens d'intervention (en progression).

Ces évolutions ont modifié les contenus et les contextes du travail ouvrier dans le sens d'un enrichissement paradoxal (source de nouvelles contraintes liées à l'autonomie d'intervention ou au rapport direct au client). «(...) les nouvelles formes de performance, y compris la réduction des coûts en situation technologiquement complexe, ont pour point commun de reposer sur des processus relationnels ouverts, sur des modes de coopération qui sont, en grande partie, en rupture avec les modes statiques et rigides de la coopération taylorienne. (...) L'idéal de non-communication entre les acteurs qui était celui du taylorisme devient clairement contreproductif 》 (Veltz, 1996).

Dans les usines où le clivage décideur/exécutant était prégnant tant sur le plan technique qu'idéologique, cette tendance a déstabilisé des identités professionnelles souvent rigides mais également les grilles de lecture des qualifications des employeurs et des salariés. Ainsi dans la nomenclature de l'INSEE : « $L a$ catégorie ouvrier est manifestement la plus chahutée par les réorganisations actuelles. Parmi les différents types de réorganisations contemporaines, la quasi-totalité trouble l'identification du travail ouvrier : la diminution du nombre de niveau hiérarchique, l'habilitation à effectuer la maintenance de premier niveau, à arrêter la production en cas d'incident, à participer à des réunions; le fait de donner des indications à des personnes extérieures à l'entreprise, de voir son rythme de travail imposé par la demande extérieure, d'être en contact avec le client ; utiliser du matériel informatique ou des NTIC (nouvelles technologies de l'information et de la communication); réaliser un entretien professionnel une fois par an. La catégorie "ouvrier" est donc celle dont l'identification est très nettement mise à mal par les mutations contemporaines de la production. » (Crague, 2003)
Les innovations technologiques et les changements organisationnels ont ainsi amené à des modifications fonctionnelles au sein de la production industrielle qui avaient été ainsi pointées dans les travaux prospectifs du Commissariat Général du Plan : "Le rôle des agents de maîtrise est amené à se réduire et à se transformer sinon à disparaître. Un nouveau personnage apparait, le conducteur de ligne: selon les branches professionnelles, ses caractéristiques oscillent entre une haute qualification technique et une compétence d'animation et de formation.» (CGP, 1999, p. 55) Mais ces transformations des emplois ouvriers, si on y ajoute le rapport au client ou les normes qualité, se sont souvent heurtées à l'absence de profils adaptés à une telle plasticité des activités mais aussi aux défaillances de la formation continue.

La constitution d'équipes «autonomes» s'est également heurtée à l'impréparation des cadres supérieurs à établir des interactions nouvelles avec les opérateurs de production: " Dès lors que disparaît tout intermédiaire entre l'équipe et un responsable de niveau élevé (qu'il s'agisse d'un agent de maîtrise ou d'un ingénieur chef de service), l'équipe perd, en quelque sorte, un fusible de transmission. Elle doit assurer sa responsabilité collective "en direct", établir, avec ce hiérarchique, un type de dialogue "gestionnaire" auquel ni lui ni elle ne sont préparés (dans la tradition managériale française)... » (Zarifian, 1998) La crise du management intermédiaire, soulignée depuis quelques années, illustre ces situations où il s'agit pour l'encadrement de reconnaître les acquis d'expérience des opérateurs dans un champ industriel fortement marqué par les traditions de clivages fonctionnels et les spécialisations techniques sectorielles. L'ode à la flexibilité, qui a entraîné une certaine volatilité de la main-d'œuvre, n'a pas non plus permis de résoudre les nouvelles exigences d'une production désormais rythmée par les démarches qualité et donc par un engagement des salariés dont les corollaires sont souvent une expérience bien trempée et une reconnaissance statutaire: "Une usine flexible, même au sens le plus courttermiste du mot, est d'abord une usine dont les processus sont fiables, bien réglés, parfaitement maîtrisés. Or cela ne va pas sans accumulation des 
compétences et des expériences et sans un minimum de stabilité des collectifs de travail. » (Veltz, 2000)

Néanmoins, malgré tous ces obstacles, les transformations de l'industrie menées avec les personnels en place ont ouvert, dans un premier temps, des espaces promotionnels pour les ouvriers (ouvrier non qualifié vers ouvrier qualifié, ouvrier qualifié vers technicien) durant la période de 1985-1993, 144000 évoluant vers une qualification plus élevée contre 22000 en régression (Chapoulié, 2000) ${ }^{4}$. Il y a donc bien eu un phénomène d'enrichissement des qualifications des ouvriers en place au début des années 80 , qui interrogeait aussi bien la faible adaptation de l'enseignement professionnel initial à cet enjeu que l'apport modeste de la formation continue des adultes: "L'élévation du niveau de formation initiale, scientifique et technique, et l'élargissement de la formation continue ont surtout concerné les mêmes catégories qu'autrefois: ingénieurs, cadres, techniciens, au mieux, une mince fraction des ouvriers hautement qualifiés ; pour la masse des salariés et des citoyens, le développement technologique important qu'a connu la société française ne s'est pas traduit beaucoup moins semble-t-il que dans d'autres pays comparables - par une croissance proportionnelle de la formation scientifique et technique de masse. Les inégalités scolaires entre les niveaux restent très importantes et la formation continue n'a pas contribué à les réduire. » (Dubar, 1982)

\section{UNE SOLLICITATION UNILATÉRALE DU SYSTĖME DE FORMATION}

Les difficultés et les réticences à associer les salariés en place aux modernisations ont amené les entreprises à considérer les jeunes "surdiplômés » (plus dociles et moins rémunérés) comme mieux adaptés aux nouvelles organisations du travail. Dans un

\footnotetext{
${ }^{4}$ La même étude de l'INSEE avait pointé un fort déplacement des ouvriers qualifiés des PME vers les grandes entreprises, soulignant même que ce flux était le plus important de l'ensemble des types de mobilité malgré une stabilité des effectifs des ouvriers artisanaux. Il reste à vérifier si ce déplacement s'explique par le potentiel de polyvalence dont étaient dotés ces salariés et qui correspondait aux nouveaux besoins des grandes unités.
}

contexte de développement du chômage, le choix de la société des années 80 fut de massifier l'enseignement secondaire et supérieur et de miser sur la montée en niveau des nouvelles générations (entre 1981 et 1995, les effectifs des BTS et DUT passeront de 67300 à 228200 soit $+239 \%$ ). Cette politique se mena au détriment d'un effort collectif ${ }^{5}$ de formation promotionnelle des salariés en place de l'industrie : "Considérant en effet que les cadres doivent être régénérés, les grandes entreprises privilégient le recrutement de diplômés supérieurs et limitent les promotions internes, y compris celles des "techniciens supérieurs" qu'elles jugent trop peu généralistes. Elles bloquent donc les promotions de ces "techniciens supérieurs" tandis qu'elles les trouvent "surdimensionnés" par rapport aux besoins effectifs de compétence qui sont les leurs pour du travail d'exécution. On comprend mieux dès lors les exclusions qui s'amorcent dans l'industrie pour les diplômés professionnels de base ainsi que l'enfermement catégoriel pour les diplômés de niveau III dès lors que leur gestion ne serait pas modifiée." (D’Iribarne, 1989) Quant au bac professionnel, censé répondre aussi aux besoins en compétences des entreprises, il découlera d'abord du mot d'ordre de « $80 \%$ d'une génération au niveau bac » plutôt qu'à la nécessité d'une refonte cohérente de l'enseignement technologique et professionnel et d'une relance de la formation continue ${ }^{6}$. Ce choix relèvera d'abord: " de la tendance très française à répondre $\grave{a}$ de nouveaux problèmes par un relèvement de niveau de recrutement plutôt que par un effort de formation du personnel en place. De ce fait, ce sont toutes les formations ouvrières qui sont dévalorisées. » (Benoist, 2000) L'enseignement professionnel de niveau $\mathrm{V}$ sera ainsi encore plus déprécié qu'auparavant avec les effets de sélection pour les jeunes de niveau $\mathrm{V}$ et les effets d'éviction pour les

\footnotetext{
${ }^{5}$ La nuance n'est pas négligeable car la politique de promotion sociale issue de la loi de 1958 comportait une dimension individuelle qui limita d'emblée son intérêt et sa portée du point de vue des syndicats. Le congé individuel de formation issu de la loi de 1971 maintiendra cette ambigüité laissant penser qu'intérêts collectifs et intérêts individuels des salariés étaient par nature antagoniques.

${ }^{6}$ Certains, au sein du système éducatif, défendirent avec raison le bac professionnel comme l'exemple à suivre d'un diplôme "promotionnel » dédié aux salariés expérimentés, mais ne furent pas écoutés.
} 
salariés en place: "L'insistance des responsables politiques sur l'élévation du "niveau" ne sera pas sans effets sur les choix des entreprises. Il paraît aller de soi que les postes les moins qualifiés sont voués à disparaître. Aussi les suppressions d'emplois toucheront-elles "naturellement" ces travailleurs dont les entreprises prévoient qu'elles n'auront plus l'usage. Et lorsque, après avoir ainsi sélectivement licencié, les entreprises embaucheront à nouveau, le pli sera pris de privilégier des critères de niveau scolaire dans les recrutements. » (Ginsbourger, 1998)

Des travaux ont, depuis, atténué les incidences des nouvelles organisations de travail en termes de niveaux de qualification, certains allant même jusqu'à émettre l'hypothèse d'un statut quo organisationnel défavorable aux nouvelles générations: «Dans la plupart des entreprises, l'automatisation des équipements de production ne s'accompagne pas d'une recomposition en profondeur des organisations du travail. La structure des qualifications reste bien souvent inchangée. Dans la mesure où l'efficacité de la transversalité suppose des organisations du travail reconfigurées où les frontières entre les fonctions de production ont bougé, cette situation est préjudiciable aux jeunes diplômés.» (Quenson, 2006)

Néanmoins, les difficultés d'ajustement entre le système de formation et les organisations du travail n'ont toujours pas été résolues et les entreprises industrielles (notamment les PME - petites et moyennes entreprises) sont confrontées à une pénurie récurrente de compétences adaptées aux nouveaux registres d'activité et aux organisations nouvelles : "L'inachèvement des profils définis par les standards (de diplômes) est particulièrement critiqué parce qu'inadapté à la situation d'une majorité d'entreprises de mécanique et de plasturgie qui utilisent des technologies largement diffusées et enseignées. Le caractère privilégié des enseignements généraux et abstraits dans la définition des référentiels et l'insuffisance accordée aux savoirs professionnels, c'est-à-dire à l'apprentissage des savoirs d'expérience au cours de la formation initiale, sont très largement déplorés (...) la majorité des diplômes de mécanique et de plasturgie (niveau $V$, IV et III) sont aux yeux des employeurs porteurs d'une information incomplète ou encore d'une partie seulement des savoirs nécessaires. (...) cette situation se manifeste principalement pour certaines activités qui concernent la transformation de la matière (mécanique, plasturgie, également chimie). Elle ne pose pas de difficultés, on l'a vu dans les domaines d'activités "artificielles" totalement issues du travail humain telle l'électricité, l'électronique, l'informatique. (...). On pourrait estimer qu'il est plus aisé pour les PME d'organiser la professionnalisation des jeunes débutants que pour les grandes entreprises. (...) Or c'est l'inverse qui se passe. L'évolution des contextes économiques des PME enquêtées (...) et les conditions concurrentielles qui sont fréquemment les leurs, particulièrement en fabrication, tendent à rendre l'apprentissage des savoirs professionnels par la pratique de plus en plus difficile. (...) Ainsi les standards adoptés en matière de diplômes professionnels et techniques se révèlent de manière dominante assez éloignés des attentes des PME industrielles. » (Campinos, Dubernet, 2000) Ces distorsions résultent de représentations qui sousestiment l'expérience et le potentiel d'apprentissage des salariés et font porter au système éducatif l'essentiel de la responsabilité de l'adéquation formation/emploi. Cette situation est venue rappeler que le diplôme "constitue une validation sociale d'acquis scolaires qui ne vaut pas reconnaissance professionnelle par le système productif » (Lochet, 2003) et qu'il ne faut donc pas lui fixer des objectifs surdimensionnés au regard de la vie professionnelle réelle.

\section{LE PÉRIMÈTRE AMBIVALENT DES DIPLÔMES PROFESSIONNELS}

La difficulté du système de formation à s'adapter aux transformations de l'industrie tient précisément au fait que celle-ci lui a imposé une subordination au modèle taylorien. Ce modèle entérinait une forte coupure entre la formation ouvrière et celle des ingénieurs. Ainsi durant toute la phase de pénurie de main-d'œuvre de l'après-guerre, la poursuite d'études a été réservée aux élites sociales et professionnelles tandis que l'on «massifiait» la sortie précoce de l'école par le biais de l'enseignement 
professionnel. Cependant, il y avait une cohérence entre le taylorisme et l'adéquationnisme des années 60 , où la «formation sur le tas » assurait une fonction corrective et intégratrice importante dans un système d'emploi stable. Les transformations organisationnelles, les avancées technologiques et l'importance des réseaux de communication auraient dû permettre une remise en cause des clivages de contenu entre enseignement professionnel et enseignement général. En fait, ces mutations ont souvent été interprétées dans la sphère éducative comme un mouvement d'intellectualisation et d'abstraction du travail qui venait en quelque sorte justifier, a posteriori, la distinction classique entre savoirs généraux et technologiques et savoirs professionnels : « $L a$ solution française qui consiste à intégrer des exigences de savoirs théoriques dans tous les diplomes professionnels, conduit à juxtaposer ce que l'on appelle le domaine technologique et professionnel et les domaines généraux. » (Bouyx, 1997) Cette conception a notamment contribué à la relégation de l'enseignement professionnel alors même que les mutations du travail permettaient de sortir des impasses du modèle scolaire.

La relance de l'apprentissage et l'émergence des formations en alternance, à la fin des années 80 , ont d'ailleurs témoigné de cette nécessité d'articuler et de mutualiser les savoirs théoriques et les savoirs pratiques. Ces modalités supposaient la transformation du contenu des diplômes professionnels et l'élargissement du périmètre d'évaluation et de certification des savoirs ainsi construits. En fait, les phases d'apprentissage en situation de travail ont insuffisamment modifié le contenu des formations dispensées dans les lieux d'enseignement et la conception des diplômes eux-mêmes. Aux niveaux V et IV, l'apprentissage et l'alternance ont plutôt conforté les conceptions adaptatives étroites de la qualification. Conceptions que les certifications de branches (CQP - certificats de qualification professionnelle) sont venues consolider au moment même où il fallait favoriser les mobilités intersectorielles et dépasser les spécificités techniques en matière de qualifications. L'enseignement supérieur court, censé répondre à l'élargissement des qualifications de la production industrielle, n'a pas pu favoriser le désenclavement de l'enseignement professionnel de niveaux $\mathrm{V}$ et IV ni d'ailleurs à la promotion ouvrière. En revanche, il a contribué paradoxalement à élargir les perspectives des élèves des filières générales et technologiques.

Cette situation renvoie à la difficulté des représentants des professions et des syndicats dans les CPC (commissions professionnelles consultatives) à développer une alternative aux représentations académiques et à rompre avec l'attachement aux particularismes technico-sectoriels indûment présentés comme des dimensions «métiers » : «(...) loin de souhaiter le développement de formations transversales aux activités de transformation des matériaux, c'est plutôt en faveur d'un renforcement des spécificités que comporte chacun de leurs métiers que se prononcent les professionnels... » (Marquette, 2004). Quant aux tentatives de certaines CPC d'expérimenter des diplômes intersectoriels comme le bac pro «pilotage de systèmes de production automatisé »(PSPA), elles ont plutôt conduit à confirmer les représentations classiques des employeurs, déroutés par des appellations généralistes au moment même où les branches élaboraient des CQP aux libellés très proches du vocabulaire des secteurs.

En ce qui concerne le caractère pluriel des compétences mobilisées par les TAM de type industriel, les référentiels de la trentaine de BTS ne sont pas avares d'objectifs ambitieux et multiples, censés être atteints par l'enseignement scolaire: "En tant que professionnel électricien, responsable d'une équipe d'intervenants et agissant souvent à l'extérieur de sa propre entreprise, (le technicien supérieur en électrotechnique) doit également développer, outre la maîtrise des aspects techniques, normatifs et réglementaires, des compétences relationnelles pour dialoguer avec les clients tant sur le plan technique que commercial. Par ailleurs, il est le garant des conditions d'exercice du métier proposées aux professionnels qu'il encadre. Il fait ainsi respecter le concept de santé et de sécurité au travail ( $S \& S T)$. L'ensemble de ces compétences lui permet de travailler en toute autonomie et de conduire une équipe d'intervenants en toute responsabilité. » (Chassaing, 2005) Ces descriptifs (en l'occurrence le BTS électrotechnique) interrogent l'amont de l'enseignement supérieur court (les jeunes qui préparent ces BTS ont-ils été préparés à ces 
dimensions ?) mais également le lien avec ce qui se construit dans le travail car l'autorité, l'autonomie ou l'expertise normative se gagnent aussi par l'expérience et correspondent à de vrais enjeux non pas seulement initiaux mais aussi promotionnels. Cette sous-estimation de l'importance des savoirs expérientiels induit chez les jeunes et chez les employeurs des représentations erronées des temporalités et des processus qui permettent de développer ces compétences d'expertise, relationnelles ou d'organisation.

Aujourd'hui, les jeunes diplômés de niveau IV ou III, ne disposent toujours pas de l'expérience concrète de l'usage des techniques en contexte de production et peinent à démontrer leur valeur ajoutée par rapport aux opérateurs moins diplômés mais expérimentés. Ce phénomène est d'autant plus prégnant que les titulaires de BTS ou de DUT ont toutes les raisons de partager le point de vue des bacheliers professionnels à l'égard des activités de production: "Pour ces jeunes gens (bacheliers professionnels) issus des spécialités orientées vers la production, il s'agissait d'échapper à la condition ouvrière, d'accéder à "une situation au-dessus de l'ouvrier", disent-ils. D'éviter, pour le moins, les aspects les plus rudes de cette condition: ne pas être condamnés à rester "derrière une machine", ne pas seulement "tourner les manivelles", comme dit un mécanicien. (...) Ils souhaitent pouvoir se tenir un peu plus loin des machines, dans des fonctions d'appui, de méthode ou de maintenance par exemple... $($ Eckert, 2008) Au sentiment de déclassement s'ajoute le constat d'un faible salaire de départ (salaire médian de $990 €$ net au premier emploi) au regard des cadres du tertiaire et d'une intégration sociale quelquefois difficile (les étudiants de BTS et de DUT viennent majoritairement des filières générales et technologiques et appartiennent plutôt à la classe moyenne).

Enfin, le moindre des paradoxes n'est pas que les emplois de TAM sont désormais "réservés» aux jeunes issus des filières générales et technologiques au détriment de ceux qui sont dans les voies professionnelles majoritairement issus des classes populaires. Ainsi, même "les apprentis du supérieur se recrutent massivement dans les lycées ou les universités. (...) $87 \%$ des apprentis de BTS sont passés par une terminale ou une formation du supérieur "à temps plein". Cette proportion est de $97 \%$ en IUT, de $98 \%$ en licence professionnelle et de $99 \%$ chez les apprentis ingénieurs » (Moreau, 2008). Le succès des DUT et BTS en matière d'insertion dans l'emploi ne doit donc pas masquer qu'ils sont porteurs de désillusions chez les jeunes déclassés mais aussi qu'ils ne répondent pas aux objectifs de promotion sociale des jeunes issus des milieux populaires et aux objectifs promotionnels des opérateurs expérimentés de l'industrie, potentiellement capables d'occuper les emplois de techniciens. Plus concrètement, ce paradoxe place d'ailleurs les syndicalistes de base dans une position très difficile face aux seuils d'embauche qui favorisent les jeunes "sur-diplômés » car, bien qu'attachés à la reconnaissance des diplômes, «ils ne peuvent défendre un principe qui consiste à qualifier un jeune bachelier professionnel à un échelon supérieur à celui d'un ouvrier moins ou pas diplômé ayant de nombreuses années d'expérience dans le métier. » (Dubernet, 2002)

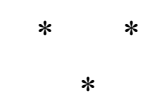

L'injonction faite au système éducatif de "courir tous les lièvres à la fois » (la sélection de l'élite, la réussite immédiate de l'insertion dans l'emploi, l'adéquation fine aux métiers, l'acquisition du maximum de connaissances générales et l'accès aux emplois les plus prestigieux) a laissé dans l'ombre les processus d'autoformation et d'apprentissage expérientiels des salariés. Mais ce choix n'a pas pour autant favorisé le rapport à l'emploi des nouvelles générations; il a même incroyablement alambiqué le parcours scolaire de jeunes de plus en plus distanciés du marché du travail : "L'empilement de certifications nationales de niveaux différents, correspondant à une seule famille de métiers, et signalant une aptitude au même métier (du CAP au BTS), est une source de confusion et d'opacité. En outre, la distinction technologique/professionnel actuellement pratiquée conduit à des impossibilités théoriques de passage d'une filière à l'autre que le système éducatif s'ingénie ensuite à rendre possible. Ainsi met-on en place des passerelles entre BAC pro et $B T S$, après avoir créé le BAC pro comme voie courte de qualification et destinée précisément à offrir une alternative au BTS. » (Fauroux, 1996) Cette situation a entériné des processus sélectifs qui n'ont cessé de 
s'aggraver: "En tout état de cause, le compromis conventionnel "à la française" n'entame guère le mécanisme de tri des élèves; en sélectionnant sur la base de critères académiques, ce tri établit une convention sociétale très puissante, à dominante méritocratique, dont la légitimité n'est guère contestée. » (Verdier, 2001)

Le « rattrapage » scolaire des années 80 a trouvé ses justifications dans l'exagération des difficultés des ouvriers peu scolarisés à s'adapter aux modernisations et l'incompréhension de leurs potentialités d'évolution. Cette stigmatisation des acquis de l'expérience a été la principale cause de la limitation de la formation promotionnelle, en France, au moment même où les conditions de son développement commençaient à être réunies. La notion d'équivalence entre savoirs scolaires et savoirs d'expérience, dans un tel contexte, loin de réhabiliter les seconds, a contribué à rendre les premiers de plus en plus inatteignables! Mais la fuite en avant " diplômante » de l'École ne résoudra ni quantitativement, ni qualitativement, le remplacement des partants du système de production. Il faudra bien se confronter à la question de la refondation des premiers et deuxièmes cycles de l'enseignement secondaire autour d'une intégration des cultures technologiques et professionnelles dans la culture scolaire, comme cela a été le cas dans d'autres pays comme l'Allemagne : "En France, les ouvriers sont surtout formés par des filières d'échec alors que les ingénieurs sont, au contraire, issus d'écoles difficiles d'accès. Il en résulte que les ouvriers français ne peuvent guère devenir techniciens ou ingénieurs, et surtout, que l'écart de qualification scolaire entre les ouvriers et les ingénieurs entraîne des différences de salaire beaucoup plus fortes qu'en Allemagne.» (Dubet, 2004)

Cette situation traduit aussi la schizophrénie des relations entre le système éducatif et les partenaires sociaux sur la construction des compétences et, donc, de la qualification professionnelle: «(...) l'espace de qualification à la française est adossé au système de classification. Il représente alors un obstacle au développement d'une véritable polyvalence et à la flexibilité, dans la mesure où cette classification met en avant des critères d'âge, d'ancienneté et de diplôme, omettant par là-même la structure de l'organisation du travail. La rigidité du modèle français s'exprime dans la confusion entretenue entre qualification et classification, renvoyant à une configuration de relations professionnelles centralisées sous l'égide de l'État. La gestion de la main-d'œuvre serait héritière d'une gestion plus administrative que professionnelle alors que les mutations du travail invitent à intégrer dans la qualification opératoire des compétences non prises en compte par la classification. L'accent mis sur le parcours de formation diplômante évacue tout recours à l'analyse des activités de travail comme variable discriminante des échelles de rémunération et réduit la compétence visible à des critères objectifs censés refléter les qualités détenues par les salariés pour occuper telle catégorie d'emplois. » (Osty, 2003)

Au lieu de se focaliser sur le début de la vie active, les systèmes d'élaboration des diplômes professionnels doivent se doter d'une approche plus large et plus dynamique de l'évolution des savoirs professionnels tant du point de vue des mobilités des personnes que des transformations du travail. Les enjeux de ces deux volets ne sont plus compatibles avec la persistance d'une conception des diplômes fondée sur le cloisonnement vertical et pyramidal, la rigidité des frontières catégorielles et fonctionnelles et la prédominance des savoirs généraux comme seuls vecteurs de la montée en qualification. Il faut resituer les ambitions de la formation professionnelle dans le champ du dialogue social, des changements du travail et des potentiels croissants des salariés qui seront de plus en plus formés, de plus en plus attentifs à leur rôle participatif et de plus en plus capables d'acquérir des compétences expertes opérationnelles. Encore faut-il que les partenaires sociaux s'emparent de ce sujet et considèrent les diplômes professionnels comme porteurs et traducteurs (de la VAE - validation des acquis de l'expérience - à la formation promotionnelle) de cette hypothèse. Une hypothèse qui suppose un recentrage des cibles d'un enseignement général, professionnel et technologique réconcilié et adapté aux organisations de travail et aux potentiels d'évolution des salariés expérimentés (qui seront par ailleurs de plus en plus dotés d'un niveau scolaire supérieur à celui des générations du baby boom). 
Benoist P. (2000), La formation professionnelle dans le Bâtiment et les Travaux Publics - 1950-1990, Paris, L'Harmattan.

Bouyx B. (1997), «Le système de négociation et de construction des diplômes technologiques et professionnels en France» in Möbus M. et Verdier E. (Eds.), Les diplômes professionnels en Allemagne et en France, Paris, L'Harmattan, pp. 52 et 53.

Campinos-Dubernet M. (2000), « Le recrutement des jeunes débutants par les PME industrielles : un difficile problème de cohérence », Cahiers $d u$ centre d'études pour l'emploi $\mathrm{n}^{\circ} 38$.

Commissariat général du plan (2002), Avenirs des métiers, Rapport du groupe « Prospective des métiers et des qualifications », La Documentation française.

Commissariat général du plan (1999), Qualifications et fonctions, une lecture transversale des contrats d'études prospectives industriels, Mission « Prospective des métiers et des qualifications », La Documentation française, p. 55.

Conseil d'analyse stratégique (2007), Les métiers à 2015, Rapport du groupe « Prospective des métiers et des qualifications », La Documentation française.

Chapoulie S. (2000), «Une nouvelle carte de la mobilité professionnelle », Revue Économie et Statistique $\mathrm{n}^{\circ} 331$, janvier.

Chassaing J.-P. (2005), «Un nouveau référentiel pour le BTS électrotechnique ", in A quoi servent les diplômes professionnels de l'Éducation nationale?, CPC info 41.

Crague G. (2003), Réorganisations et déstabilisation de la qualification, LATTS ENPC - contribution aux journées « changements organisationnels et informatisation (COI) » des 4-5 décembre 2003.

Dauty F. Lemistre Ph. et Vincens J. (2006), «La nomenclature des niveaux de formation, sens, portée, devenir », CPC documents, $\mathrm{n}^{\circ} 2$, pp. 58 et 59.
Dubar C. (1982) « Technologies nouvelles, transformations du travail et besoins de formation », Revue POUR $\mathrm{n}^{\circ} 85$, septembre.

Dubernet A.-C. (2002), « Des métiers traditionnels aux vrais métiers" in Piotet F. La révolution des métiers, PUF.

Dubet F. (2004), L'école des chances. Qu'est-ce qu'une école juste?, Seuil, "La République des idées ».

Eckert H. (2008), « Quelle carrière pour les titulaires d'un Bac professionnel?»- hors série pratique Alternatives Économiques - L'état de l'emploi.

Fauroux R. (1996), Pour l'école, rapport au Premier ministre, Paris, Fayard.

Flacher D. et Pelletan J. (2007), « Le concept d'industrie et sa mesure: origines, limites et perspectives. Une application à l'étude des mutations industrielles », Économie \& Statistique ${ }^{\circ}$ 405/406.

Ginsbourger F. (1998), La gestion contre l'entreprise, Paris, La Découverte.

D’Iribarne A. (1989), La compétitivité, Paris, Éditions du CNRS.

Lochet J.-F. (Coord.) (2003), Entreprises et jeunes débutants, Céreq et L'Harmattan.

Marquette C. (2004), « Nouveaux savoirs, structuration des enseignements et organisation des secteurs professionnels : quelles relations ? Le cas des activités industrielles de mise en œuvre/transformation des matériaux », document Céreq, juillet.

Moreau G. (2008), «Apprentissage : une singulière métamorphose », Revue Formation Emploi ${ }^{\circ} 101$, janvier-mars.

Osty F. (2003), Le désir de métier, engagement, identité et reconnaissance au travail, Presses Universitaires de Rennes. 
Quenson E. (2006), « La transversalité des diplômes de la production : un modèle de certification reconnu sur le marché du travail?» in Les diplômes de l'Éducation nationale dans l'univers des certifications professionnelles, nouvelles normes et nouveaux enjeux, CPC documents $\mathrm{n}^{\circ} 3$.

Stroobants M. (1993), Savoir-faire et compétences au travail, Éditions de l'université de Bruxelles.

Tanguy L. (Dir.) (1986), L'introuvable relation formation/emploi, Paris, La Documentation française.
Verdier É. (2001), «La France a-t-elle changé de régime d'éducation et de formation?» in Numéro spécial " 30 ans d'analyses», Revue Formation Emploi, Céreq, octobre-décembre.

Veltz P. (1996), Mondialisation, villes et territoires, l'économie d'archipel, Paris, PUF.

Veltz P. (2000), Le nouveau monde industriel, Paris, Gallimard, pages 131 et 197.

Zarifian Ph. (1998), Travail et communication, $2^{\mathrm{e}}$ édition, Paris, PUF.

\section{Résumé}

\section{Les distorsions diplômes/qualifications: \\ l'exemple des techniciens et agents de maîtrise de type industriel}

\section{Paul Santelmann}

Les distorsions constatées depuis plusieurs décennies entre l'organisation du système éducatif et le redéploiement des qualifications ne tiennent pas seulement à la difficulté des nomenclatures à cerner les modifications de la division du travail. La montée en niveau des diplômes s'est imposée comme une réponse d'évidence face à des mutations du système de production essentiellement appréhendées sous l'angle de l'intellectualisation du travail. Cette conception, centrée sur une surestimation du rôle de la formation initiale, a entraîné à la fois des processus de dévalorisation et d'éviction des salariés en place et des phénomènes de sur-sélection à l'embauche et de déclassement des jeunes. Le polymorphisme des techniciens et des agents de maîtrise (TAM) de l'industrie illustre le décalage entre les objectifs assignés au système éducatif et la production des compétences et des qualifications dans les activités de travail.

\section{Mots clés}

Technicien, agent de maîtrise, profession intermédiaire, secteur secondaire, relation formation-emploi Journal of Economic Literature: J 29 